\title{
Edg-1, the G protein-coupled receptor for sphingosine-1-phosphate, is essential for vascular maturation
}

\author{
Yujing Liu, ${ }^{1}$ Ryuichi Wada, ${ }^{1}$ Tadashi Yamashita, ${ }^{1}$ Yide $\mathrm{Mi},{ }^{1}$ \\ Chu-Xia Deng, ${ }^{1}$ John P. Hobson, ${ }^{2}$ Hans M. Rosenfeldt, ${ }^{2}$ \\ Victor E. Nava, ${ }^{2}$ Sung-Suk Chae, ${ }^{3}$ Menq-Jer Lee, ${ }^{3}$ \\ Catherine H. Liu, ${ }^{3}$ Timothy Hla, ${ }^{3}$ Sarah Spiegel, ${ }^{2}$ and Richard L. Proia ${ }^{1}$ \\ ${ }^{1}$ Genetics of Development and Disease Branch, National Institute of Diabetes and Digestive and Kidney Diseases, \\ NIH, Bethesda, Maryland, USA \\ ${ }^{2}$ Department of Biochemistry and Molecular Biology, Georgetown University Medical Center, Washington, DC, USA \\ ${ }^{3}$ Center for Vascular Biology, Department of Physiology, University of Connecticut Health Center, \\ Farmington, Connecticut, USA \\ Address correspondence to: Richard L. Proia, Building 10, Room 9N-314, National Institutes of Health, \\ 10 Center DR MSC 1821, Bethesda, Maryland 20892-1821, USA. Phone: (301) 496-4391; \\ Fax: (301) 496-9878; E-mail: proia@nih.gov.
}

Received for publication July 28, 2000, and accepted in revised form September 12, 2000.

Sphingolipid signaling pathways have been implicated in many critical cellular events. Sphingosine-1-phosphate (SPP), a sphingolipid metabolite found in high concentrations in platelets and blood, stimulates members of the endothelial differentiation gene (Edg) family of G protein-coupled receptors and triggers diverse effects, including cell growth, survival, migration, and morphogenesis. To determine the in vivo functions of the SPP/Edg signaling pathway, we disrupted the Edg1 gene in mice. Edg1-/- mice exhibited embryonic hemorrhage leading to intrauterine death between E12.5 and E14.5. Vasculogenesis and angiogenesis appeared normal in the mutant embryos. However, vascular maturation was incomplete due to a deficiency of vascular smooth muscle cells/pericytes. We also show that Edg-1 mediates an SPP-induced migration response that is defective in mutant cells due to an inability to activate the small GTPase, Rac. Our data reveal Edg- 1 to be the first $\mathrm{G}$ protein-coupled receptor required for blood vessel formation and show that sphingolipid signaling is essential during mammalian development.

J. Clin. Invest. 106:951-961 (2000).

\section{Introduction}

Sphingolipids have emerged as important signaling molecules in a variety of biologic processes (1-3). SPP in particular has come to the fore as a mediator of an extracellular signaling pathway through its interaction with the family of $\mathrm{G}$ protein-coupled receptors known by the acronym, Edg (endothelial differentiation gene) (4). Edg-1, the first of these receptors described, was identified as a gene induced during human endothelial cell differentiation (5). Activation of the Edg receptors triggers diverse effects including proliferation, survival, migration, morphogenesis, adhesion molecule expression, and cytoskeletal changes and has led to the view that the Edg receptor signaling pathways may have important roles in many physiological and pathological events (reviewed in refs. 6-10).

The Edg family can be subdivided into either receptors for SPP or for lysophosphatidic acid. The Edg receptors for SPP activate different and sometimes overlapping $\mathrm{G}$ protein-mediated intracellular signaling pathways. For instance, Edg- 1 couples directly to the $G_{i}$ pathway, whereas Edg- 3 and -5 stimulate $G_{i}, G_{q}$, and $\mathrm{G}_{13}$ pathways with differing degrees of potency (4, 11-14). Moreover, the expression pattern of individual Edg receptors changes during development and differentiation, leading to different combinations on cells and tissues (15-18). The diverse receptor expression and activation of divergent signaling pathways may explain the pleiotropic responses to SPP but have made functional analysis difficult.

To determine the functions of the SPP/Edg-1 signaling pathway, we have disrupted Edg1 in mice. Homozygous Edg1 mutant mice die in utero due to massive embryonic hemorrhage. They undergo normal vasculogenesis and angiogenesis but are severely impaired in vessel maturation due to a defect in the recruitment of mural cells to vessel walls. The results reveal the SPP receptor Edg-1 as mediating a novel $G$ protein-coupled signaling pathway required for blood vessel development.

\section{Methods}

Generation of Edg1 mutant mice. To generate the Edg1 knockout mice, we cloned a 10-kb genomic DNA fragment containing the entire Edg1 gene from a 129/Sv 
library. As shown in Figure 1a, the Edg1 gene is composed of two exons and an intron (16). The second large exon contains a $5^{\prime}$-UTR region, the entire open reading frame region, and approximately $1.8 \mathrm{~kb}$ of the $3^{\prime}$-UTR region. For knocking-in the $L a c Z$ reporter gene and targeted inactivation of the Edg1 gene, a NcoI site in the beginning of the $E d g 1$ open reading frame was used to insert a LacZ-neo (neomycin-resistant gene) cassette (19). In the construct used for disruption of the Edg1 gene, the $L a c Z$ coding region is preceded by an internal ribosomal entry sequence (20). Therefore, targeted insertion generates a bi-cistronic transcription unit in which the expression of the $\beta$-galactosidase reporter protein is under the control of $E d g 1$ transcriptional regulatory elements. The herpes simplex virus thymidine kinase (TK) gene was located outside the homologous sequence to prevent random integration.

Gene targeting in TC1 embryonic stem (ES) cells and generation of chimeric and heterozygous mice were as described previously (21). One targeted ES clone was used to establish chimeric mice, which were crossed with C57BL/6 mice to obtain Edg1 heterozygotes. All mice analyzed were obtained from intercrosses of the Edg1 heterozygotes. Edg1 genotypes were determined by Southern blot and PCR analyses of genomic DNA isolated from ES cells, yolk sacs and tail biopsies. For genotyping by PCR, the primers were: 5'TAGCAGCTATGGTGTCCACTAG3' (Primer 1), 5'GATCCTGCAGTAGAgGatgGC3' (Primer 2), 5'TtGGagtgaCgGCagtTatctgGa3' (Primer 3), and 5'TCAACCACCGCACGATAGAGATTC3' (Primer 4). Primers 1 and 2 detected the wild-type Edg1 allele and amplified an approximately 630-bp fragment. Primers 3 and 4 detected the Edg $1^{L a c Z}$ allele and amplified an approximately $350-\mathrm{bp}$ fragment. Forty-five cycles of $94^{\circ} \mathrm{C}(1$ minute $), 55^{\circ} \mathrm{C}(1$ minute $)$, and $72^{\circ} \mathrm{C}$ (3 minutes) were used.

Histological analysis. Embryos at embryonic days (E) 9.5-16.5 were removed from the mother after heterozygous mating. Then the embryos were fixed and processed to be embedded in paraffin. Serial sections (5- $\mu \mathrm{m}$-thick) were made at $15-\mu \mathrm{m}$ intervals and stained with hematoxylin and eosin (H\&E).

Paraffin sections were deparaffinized and rehydrated. Antigen retrieval was accomplished by 30 -minute incubation at $95^{\circ} \mathrm{C}$ in Target Retrieval Solution (DAKO Corp., Carpenteria, California, USA). Endogenous peroxidase activity was quenched by incubation with $5 \%$ hydrogen peroxide in methanol for 5 minutes. Specimens were incubated with anti-smooth muscle $\alpha$ actin (EPOS anti-SM $\alpha$ A/HRP; no. U7033; DAKO Corp.) for 1 hour at room temperature. After washing with PBS, peroxidase reaction was visualized with diaminobenzidine/hydrogen (DAB/hydrogen) peroxide.

To define the developmental and tissue-specific expression patterns of Edg1 through X-Gal staining, embryos dissected out from the decidua at various developmental stages were fixed in $2 \%$ formaldehyde $2 \%$ glutaraldehyde in PBS for 10 minutes. They were washed in PBS and then incubated in PBS containing $5 \mathrm{mM} \mathrm{K}_{3} \mathrm{Fe}(\mathrm{CN})_{6}, 2 \mathrm{mM} \mathrm{MgCl}_{2}$, and $1 \mathrm{mg} / \mathrm{ml}$ $\mathrm{X}-\mathrm{Gal}$ at $37^{\circ} \mathrm{C}$ overnight. Reactions were stopped by rinsing embryos with PBS, followed by further fixation in $4 \%$ paraformaldehyde.

Whole-mount embryo immunostaining. Embryos were dissected out and fixed in $4 \%$ paraformaldehyde in PBS at $4^{\circ} \mathrm{C}$ overnight. They were then dehydrated through a methanol series and stored in 100\% methanol at $-20^{\circ} \mathrm{C}$. The embryos were bleached in $6 \%$ hydrogen peroxide/methanol for 1 hour at room temperature and rehydrated through a methanol series to PBS + 0.1\% Tween 20 (PBST). They were incubated in a blocking solution (4\% BSA in PBST) twice, for 1 hour each time. The embryos were incubated with rat mAb's (anti-PECAM-1: no.1951D; anti-CD34: no. 09431D; anti-VE-cadherin: no. 28091D; PharMingen, San Diego, California, USA), diluted 1:200 in 10\% goat serum and $4 \% \mathrm{BSA}$ in PBST at $4^{\circ} \mathrm{C}$ overnight. Embryos were washed with $4 \%$ BSA in PBST at room temperature and then incubated with peroxidase-conjugated goat anti-rat Ig in $10 \%$ goat serum and $4 \%$ BSA in PBST at $4^{\circ} \mathrm{C}$ overnight. Peroxidase reaction was visualized with $\mathrm{DAB} /$ hydrogen peroxide.

RT-PCR and immunoblotting. Total RNA was isolated from E12.5 mouse embryos and cultured cells using Trizol (Life Technologies Inc., Gaithersburg, Maryland, USA) and treated with DNaseI (Life Technologies). Total RNA $(5 \mu \mathrm{g})$ was reverse transcribed using Superscript Preamplification System (Life Technologies) according to the manufacturer's instructions. PCR was performed on $2 \mu \mathrm{l}$ of the RT reaction in a volume of 50 $\mu \mathrm{l}$ using AmpliTaq Gold polymerase (Perkin-Elmer Corp., Norwalk, Connecticut, USA). The PCR conditions were as follows: initial denaturation at $95^{\circ} \mathrm{C}$ for 10 minutes followed by up to 35 cycles of denaturation at $95^{\circ} \mathrm{C}\left(1\right.$ minute), annealing at $55^{\circ} \mathrm{C}(1$ minute), and extension at $72^{\circ} \mathrm{C}(1 \mathrm{~min})$. Amplified PCR products were analyzed by electrophoresis on a $2 \%$ agarose gel. PCR primer pairs were as follows:

Flt-1: (5'TGTGGAGAAACTTGGTGACCT3', 5'TGGAGAACAGCAGGACTCCTT3') Flk-1: (5'TCTGTGGTTCTGCGTGGAGA3'; 5'GTATCATTTCCAACCACCCT3')

Tie-1: (5'TCTTTGCTGCTCCCCACTCT3', 5'ACACACACATTCGCCATCAT3')

Tie-2: (5'CCTTCCTACCTGCTACTTTA3', 5'СCACTACACСТTТCTTTACA3')

Ang-1: (5'AAGGGAGGAAAAAGAGAAGAAGAG3', 5'GTTAGCATGAGAGCGCATTTG3)

Ang-2: (5'TGCCTACACTACCAGAAGAAC 3', 5'TATTTACTGCTGAACTCCCAC 3')

PECAM-1: (5'GTCATGGCCATGGTCGAGTA3', 5'CTCCTCGGCATCTTGCTGAA3')

VE-cadherin: (5'GGATGCAGAGGCTCACAGAG3', 5'CTGGCGGTTCACGTTGGACT3')

Smad5: (5'CTTTCCACCAACCCAACAAC3', 5'TCATAGGCGACAGGCTGAAC3') endoglin: (5'TACTCATGTCCCTGATCCAGCC3', 
5'GTCGATGCACTGTACCTTTTTCC3')

LKLF: (5'CCACACATACTTGCAGCTACAC3',

5'CCATCGTCTCCCTTATAGAAATA3')

Edg-1: (5'TAGCAGCTATGGTGTCCACTAG-3',

5'GATCCTGCAGTAGAGGATGGC3')

For immunoblotting, detergent extracts of E12.5 embryos were analyzed for protein expression using antibodies against the following proteins: VE-cadherin (catalog no. 28091D; PharMingen); N-cadherin (catalog no. SC-7939; Santa Cruz Biotechnology Inc., Santa Cruz, California, USA); and P-cadherin (catalog no. SC7893), PDGF-B (catalog no. SC-7878), and VEGF (catalog no. SC-507; all from Santa Cruz Biotechnology). A peroxidase-conjugated secondary antibody was used, and the reaction was visualized with the ECL + Plus Western blotting system (Amersham Pharmacia Biotech, Piscataway, New Jersey, USA).

In vitro assays. Chemotactic migration of cells was measured in a modified Boyden chamber as described previously using polycarbonate filters $(25 \times 80 \mathrm{~mm} ; 12$ $\mu \mathrm{m}$ pore size $)$ coated with collagen type I $(50 \mu \mathrm{g} / \mathrm{ml}$ in $5 \%$ acetic acid), which promotes uniform attachment to and migration across the filter without formation of a barrier (22). SPP or medium without serum was placed in the lower chamber as chemoattractants. Mouse embryonic fibroblasts were harvested and added to the upper chamber at $5 \times 10^{4}$ cells per well. Each data point was the average number of cells in four random fields, each counted twice. Each determination represents the average \pm SD of three individual wells. For the detection of GTP-bound activated Rac, embryonic fibroblasts were serum-starved for 24 hours before they were treated with SPP (200 nM) for 5 minutes. The cell lysates were used for affinity precipitation with the PAK-1 p21 binding domain-conjugated (PDB-conjugated) agarose beads (Upstate Biotechnology Inc., Lake Placid, New York, USA) as described elsewhere (23), or were used without fractionation to determine total Rac levels. Rac was visualized by immunoblotting with an mAb.

\section{Results}

Generation of Edg1 knockout mice with an inserted $\beta$-galactosidase (LacZ) reporter gene. Edg1 consists of two exons (16), with the entire coding region in the second exon (Figure 1a). To disrupt Edg1 in mouse ES cells, we constructed a replacement-type targeting vector in which the Edg1 coding region, containing 382 amino acids, was disrupted after the first 42 amino acids by a LacZNeo cassette containing a internal ribosome entry sequence (19). This targeting strategy should both create a disrupted Edg1 allele and enable analysis of Edg1 expression in mice by creation of an Edg1-LacZ hybrid transcript driven by the endogenous Edg-1 promoter elements. The targeting vector was linearized and electroporated into the TC-1 ES cell line. Genomic DNA from G418- and ganciclovir-resistant clones was analyzed by Southern blotting. Of the 120 clones examined, about $60 \%$ contained the $2.5-\mathrm{kb}$ BamHI band diagnostic of a homologous recombina- tion event. Targeted ES cells were injected into C57BL/6 mouse blastocysts and produced highly chimeric male mice that transmitted the targeted allele through the germ line (Figure 1b). No Edg $1^{\text {LacZ }}$ homozygotes were found among more than 100 newborn animals from heterozygous crosses (Table 1), indicating an embryonic lethal phenotype. Heterozygous Edg1 mice appeared at the expected frequency and were phenotypically normal.

Edg1 ${ }^{\text {Lac } Z}$ homozygous embryos were present in utero as determined by Southern analysis. To confirm that Edg1 was disrupted by the targeting scenario, RT-PCR was performed on total embryonic RNA using primers flanking the disrupted sequence (RT-5' and RT- $3^{\prime}$ in Figure 1a). The predicted 630 -bp product was amplified from RNA of both wild-type and heterozygous embryos (Figure 1c). No PCR amplified band was detected from RNA of homozygous mutant embryos, indicating disruption of the normal Edg1 transcripts.

Expression patterns of Edg1 gene during mice embryogenesis. LacZ expression, under the control of native Edg1 regulatory elements, was visualized by X-Gal staining in heterozygous embryos. At E9.5, intense expression was observed in the common ventricular chamber of heart, dorsal aorta, intersomitic arteries, and capillaries (Figure 1d). Weak expression was detected in the forebrain and common atrial chamber of the heart. Very weak or no expression was found in the anterior and posterior cardinal veins (Figure $1 \mathrm{~d}$ ). In addition to the expression pattern observed in the E9.5 embryo, E10.5 embryos showed prominent LacZ expression in the forebrain and a weaker level of expression in the spinal cord (Figure 1e). To examine the expression of the Edg1 gene in detail, histological sections of X-Gal stained embryos were evaluated. The expression was most prominent in the endothelial cells of arteries and capillaries (Figure 1f), cardiomyocytes, and neuronal cells of the telencephalon (data not shown). No expression was found in the endothelial cells of veins (Figure $1 \mathrm{~g})$. Low levels of expression were detected the smooth muscle layers surrounding the aorta (Figure 1h).

Characterization of Edg1 $1^{-/}$embryos. To determine the time of embryonic lethality, embryos at various stages of gestation were isolated. Genotyping of E9.5 to E11.5 embryos from Edg1 heterozygous intercrosses

Table 1

Genotype analysis of offspring from Edg1 heterozygous intercrosses

\begin{tabular}{|c|c|c|c|c|}
\hline Age & Total & $\begin{array}{c}\text { Genotype } \\
+/+\end{array}$ & $\begin{array}{c}\text { Genotype } \\
+/-\end{array}$ & $\begin{array}{c}\text { Genotype } \\
-/-\end{array}$ \\
\hline E9.5 & 74 & 19 & 31 & 24 \\
\hline E10.5 & 42 & 8 & 23 & 11 \\
\hline E11.5 & 30 & 8 & 14 & 8 \\
\hline E12.5 & 113 & 32 & 55 & $26^{A}$ \\
\hline E13.5 & 45 & 9 & 28 & $8^{A}$ \\
\hline E14.5 & 33 & 12 & 21 & 0 \\
\hline E15.5-16.5 & 35 & 12 & 23 & 0 \\
\hline Adult & 115 & 38 & 77 & 0 \\
\hline
\end{tabular}

AHemorrhage. 
revealed inheritance of the $E d g 1$ mutant allele at the expected mendelian frequency (Table 1). Up to E11.5, $E d g 1^{-/-}$embryos appeared phenotypically normal. At E12.5, the $E d g 1^{-/-}$embryos could be identified by their abnormal yolk sacs, which were edematous, with less blood in the otherwise normal looking, highly branched, vasculature (Figure 2a, arrows). After removing the yolk sac, intraembryonic bleeding was evident in the $E d g 1^{-/-}$embryos (Figure $2 \mathrm{~b}$ ). The pericardial cavity of mutant embryos was enlarged and filled with fluid. The limbs of mutant embryos were underdeveloped and rounded with areas of bleeding. In comparison, age-matched wild-type embryos had more developed, fan-shaped limbs. Much less blood was found in the yolk sac blood vessels of E13.5 $E d g 1^{-/-}$embryos compared with those of the mutant embryos obtained 1 day earlier (Figure 2c, arrows). At E13.5, massive intraembryonic bleeding could be observed through the yolk sac. In addition to widespread hemorrhage, severe edema was observed
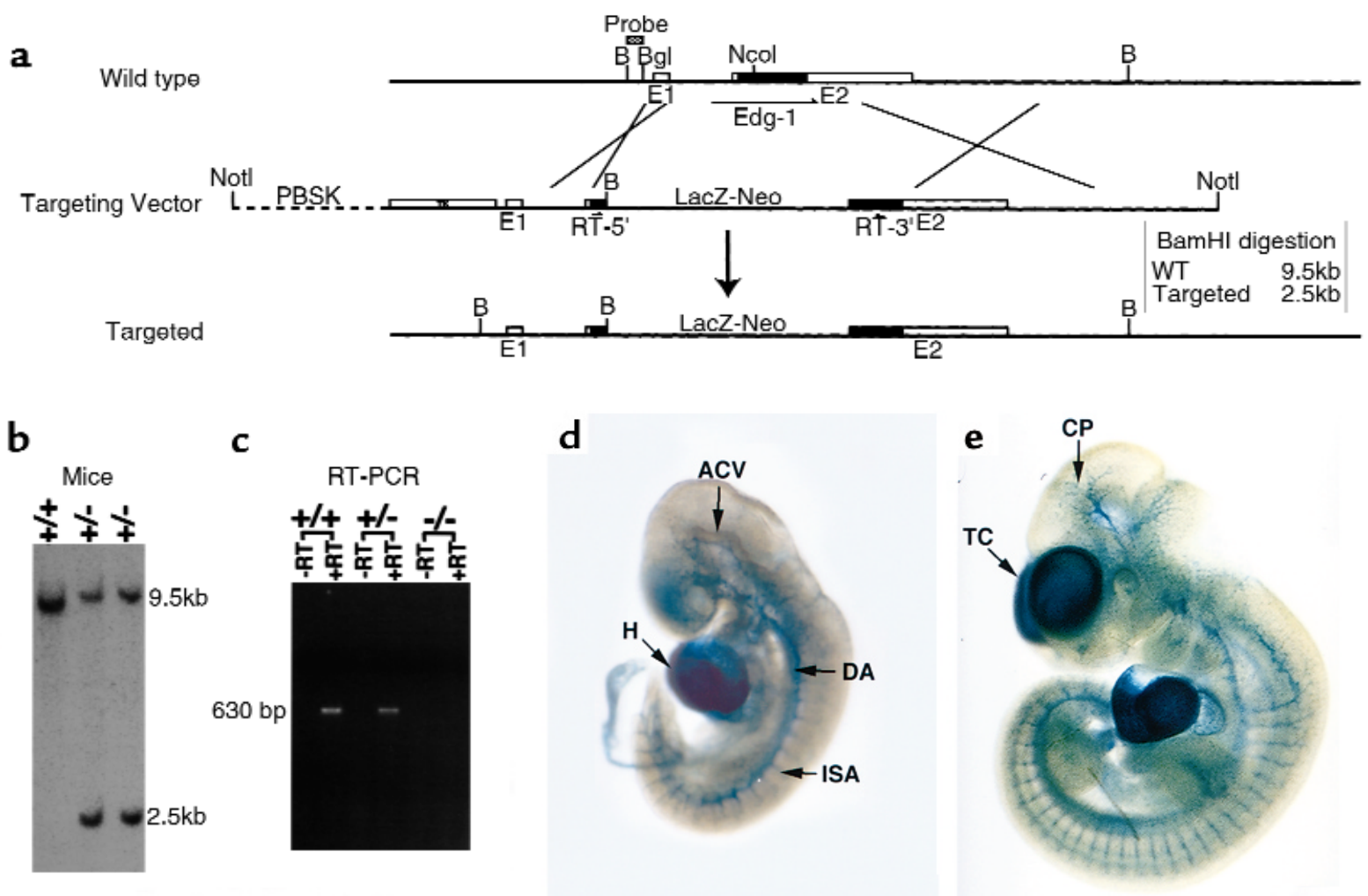

E9.5
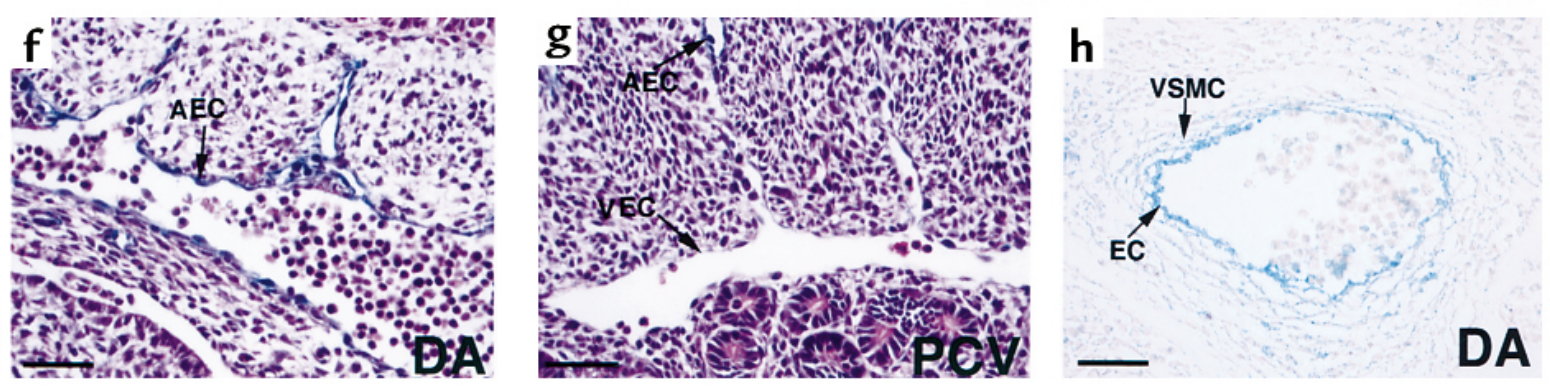

Figure 1

Targeted disruption and embryonic expression of the Edg1 gene. (a) Schematic representation of the Edg1 targeting strategy. The structure of the mouse Edg1 locus is shown at the top, the structure of the Edg1 targeting vector in the middle, and the predicted structure of the homologous recombined locus on the bottom. RT-5' and RT-3', primers for RT-PCR. B, BamHI; BgI, BgIII; PBSK, pBluescript vector. (b) Genotyping of mouse offspring from the Edg1 heterozygous mating. Wild-type Edg1 locus yielded a 9.5-kb BamHI band. Disrupted Edg1 locus yielded a 2.5-kb BamHI band. No Edg1-/- mice were found born alive. (c) RT-PCR analysis of total RNA from E12.5 mouse embryos by using RT-5' and RT-3'. Edg1 $1^{+/+}$and $E d g 1^{+/-}$RNA yielded the predicted 630-bp amplification product. No amplification product was detected from Edg1-/- RNA. (d, e) Whole-mount of Edg1 $1^{+/-}$E9.5 and E10.5 embryos stained with X-Gal. H, heart; DA, dorsal aorta; ISA, intersomatic arteries; CP, capillaries; TC, telencephalon; ACV, anterior cardinal vein. (f) Longitudinal section of dorsal aorta (DA) from E10.5 Edg1 ${ }^{+/-}$embryo. LacZ staining is seen in arterial ECs (AEC). (g) Longitudinal section of posterior cardinal vein (PCV) from E10.5 Edg $1^{+/-}$embryo. LacZ staining is seen in arterial endothelial cells (AEC) but not in venous endothelial cells (VEC). (h) Transverse section of dorsal aorta from E12.5 Edg $1^{+/-}$embryo. Vascular ECs and VSMCs are stained. EC, endothelial cell; VSMC, vascular smooth muscle cell. Scale bars $=50 \mu \mathrm{m}$. 


\section{Figure 2}

Phenotype of Edg1-/- embryos and normal vascular network in the Edg1-/- embryos. Photomicrographs of E12.5 and E13.5 embryos with the amnion, yolk sac, and placenta intact (a and $\mathbf{c}$ ), or with extraembryonic membranes removed (b and d). Edg1-/embryos show normal yolk sac vasculature but with less blood (arrows). Yolk sacs of $E d g 1^{-1-}$ embryos display progressive edema. E12.5 Edg1-/- embryo shows intraembryonic hemorrhages in the body and limbs. FL, front limb; HL, hind limb. E13.5 Edg1-/embryo demonstrates severe intraembryonic hemorrhages and edema. Both E12.5 and E13.5 Edg1 $1^{-/-}$embryos display pericardial cavity (PCC) edema. (e-h) E12.5 wild-type and Edg1-/- embryos were stained with an anti-CD34 $\mathrm{mAb}$ and visualized by low-power (e and $\mathbf{f}$ ) or higher-power ( $\mathbf{g}$ and $\mathbf{h}$ ) magnification. Note the normal vascular patterning, capillary plexus, and capillary sprouting (black arrowheads) in the Edg1-1embryos. Small blood vessels in the

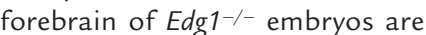
slightly dilated (white arrowheads).
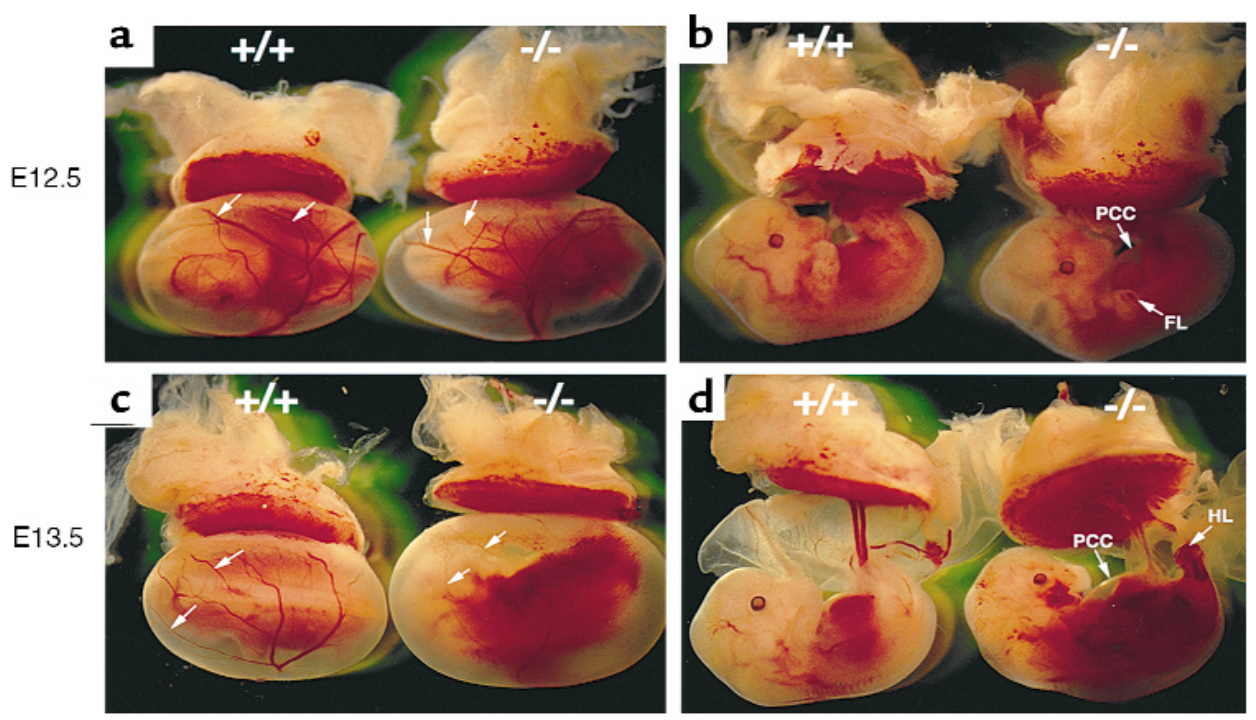

\footnotetext{
E12.5
}
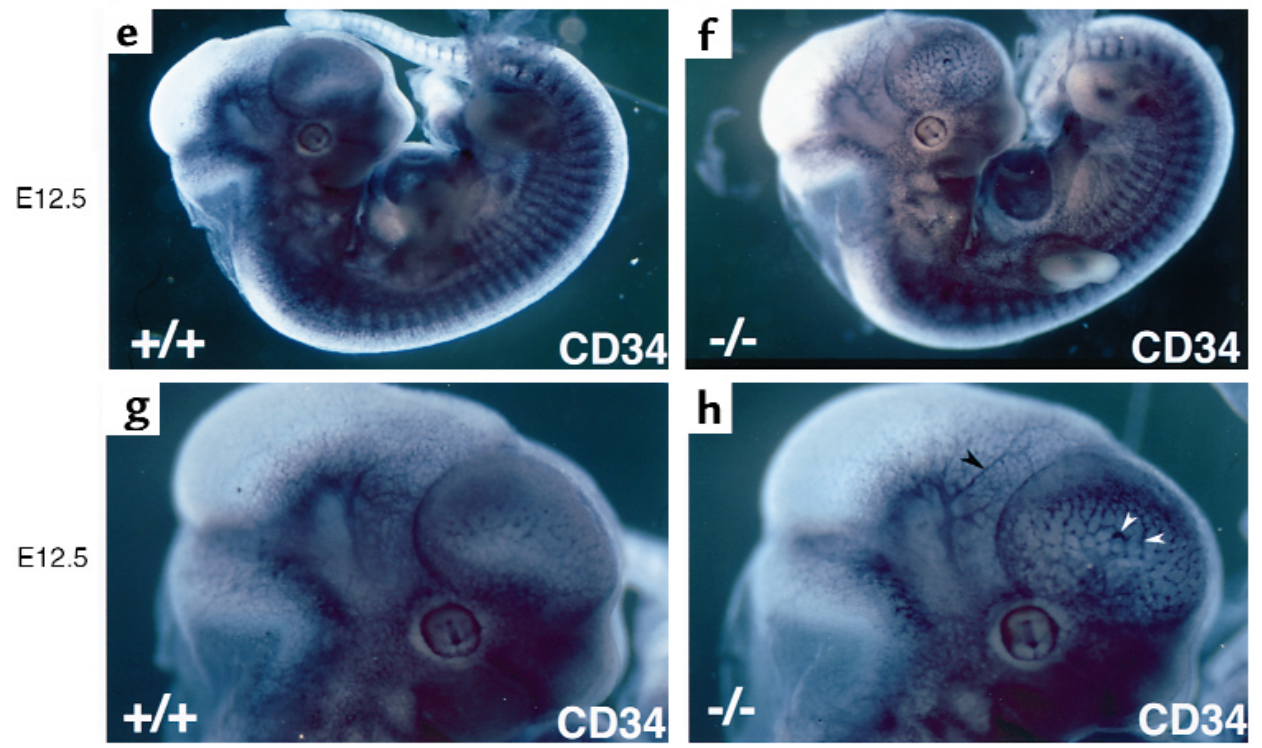

throughout the body of $E d g 1^{-/-}$embryos (Figure 3d). No Edg1 $1^{-/-}$embryos survived beyond E14.5 (Table 1).

Normal vasculogenesis in Edg1-/- embryos. To define the vascular system in the $E d g 1^{-/-}$embryos, the morphology of the vasculature was characterized by wholemount immunohistochemical staining using mAb's against markers for endothelial cells. Antibodies to CD34 (Figure 2, e and f) and platelet endothelial cell adhesion molecule-1 (PECAM-1) (data not shown) revealed a substantially normal arborized vascular network both in the mutants and age-matched control embryos. High magnification views showed capillary sprouts in the head of mutant embryos (Figure 2, g and $h$, black arrowhead). However, the small vessels in the forebrain of mutant embryos appeared dilated and stained darker than controls with antibodies against PECAM-1 and CD34 (white arrowheads).

Because SPP signaling through Edg-1 was shown to be involved in adherens junction assembly in HUVEC cells in vitro (24), we investigated the expression of VE-cad- herin, P-cadherin, E-cadherin, $\mathrm{N}$-cadherin, and PECAM1 in Edg1-/- embryos by using whole-mount immunohistochemistry and Western blot analysis. No obvious difference was observed in expression patterns between mutant and control embryos. Disruption of VE-cadherin function causes increased endothelial apoptosis (25); however, there was no apparent enhancement of endothelial cell death in E12.5 Edg1 $1^{-/-}$embryos as determined by TUNEL staining (data not shown).

Because Edg1 is highly expressed in vascular endothelial cells, endothelial cell proliferation and differentiation signaling factors, including VEGF, Flt1, Flk-1, Tie-1, Tie-2, Ang-1, and Ang-2, were analyzed by Western blot and RT-PCR methods. All of these genes were expressed in $E d g 1^{-/-}$embryos at levels similar to those of control embryos (data not shown). Thus the expression of genes required for early differentiation and assembly of endothelial cells into the vascular network was not impaired in the Edg1 $1^{-/-}$embryos. Our data indicate that in $E d g 1^{-/-}$embryos, vasculogenesis 


\section{Figure 3}

Vascular smooth muscle defects in the $E d g 1^{-/-}$ embryos. (a and b) Aortae of E12.5 embryos, sectioned longitudinally and stained with anti-SM $\alpha A$ antibody. Note the lack of SM $\alpha$ A-positive smooth muscle cells (bracket) on the dorsal side of aorta in the Edg1 $1^{-/}$embryo. (c and d) Transverse sections of aortae from E12.5 embryos stained with antiSM $\alpha A$. Smooth muscle cells have accumulated at the ventral site of the aorta in the Edg1/-- embryo. Note the discontinuous endothelial cell (EC) layer (bracket) in the Edg1 $1^{-/}$embryo. Many blood cells have leaked out to the surrounding tissues in the Edg1 $1^{-/}$embryo (arrowheads). BC, blood cells. (e and $\mathbf{f}) \mathrm{H} \& \mathrm{E}$ staining of aorta from E11.5 and 12.5 Edg1-/- embryos. Arrows point to ECs. Note their normal, flattened morphology in e and abnormal, cuboidal morphology in $\mathbf{f}$. ( $g$ and $\mathbf{h}$ ) Cranial arteries from E12.5 embryos stained with anti-SM $\alpha$ A antibody. Note the clustering of smooth muscle cells and nearly naked endothelial tube from the Edg1-/- embryo. (i and $\mathbf{j}$ ) Sections of intestine from E12.5 embryos stained with anti-SM $\alpha$ A. Note that that coverage of intestine by smooth muscle is similar in control and mutant embryos. Scale bars $=1$ $\mathrm{mm}$ ( $\mathbf{a}$ and $\mathbf{b}$ ); $50 \mu \mathrm{m}$ (c and $\mathbf{d}$ ); $50 \mu \mathrm{m}$ (e and $\mathbf{f}$ ); $50 \mu \mathrm{m}$ ( $\mathbf{g}$ and $\mathbf{h}) ; 500 \mu \mathrm{m}$ (i and $\mathbf{j})$.
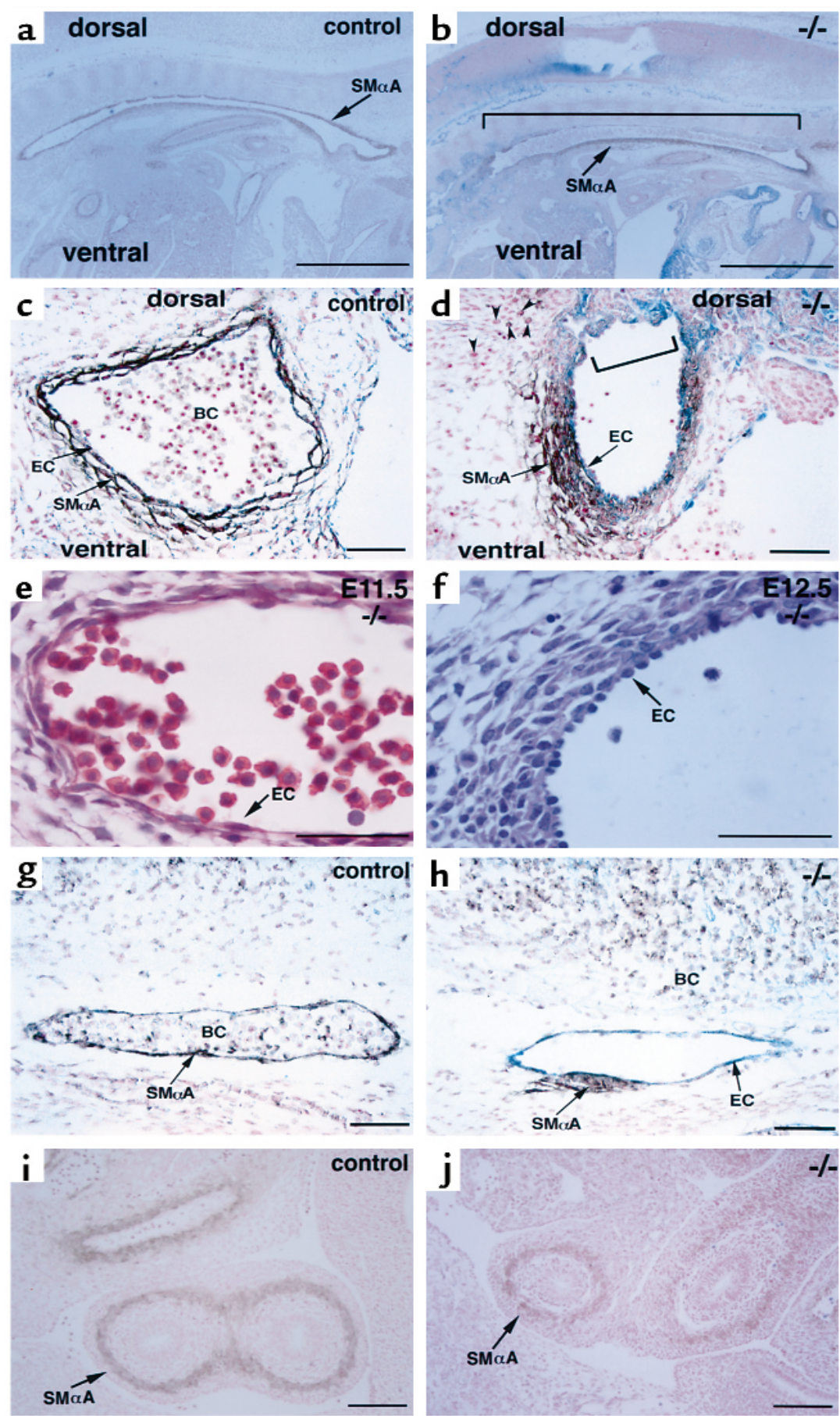

and the phase of angiogenesis that entails vessel sprouting and penetration had occurred.

Vascular smooth muscle defects in Edg1 $1^{-/}$embryos. After the initial formation of the vascular plexus, vessels mature by the stabilization of the endothelial vascular network through a recruitment and differentiation process that ultimately results in the investment of vessel walls with mural cells (26).

Vascular smooth muscle cells (VSMCs) first appear on the ventral side of the aorta in E10.5 embryos, followed by migration to the dorsum (27). By E11.5, the aorta is completely enveloped by VSMCs (27). To assess this aspect of vessel development in the Edg1 mutant embryos, VSMCs were identified using an antibody to SM $\alpha$ A. In longitudinal sections of E12.5 control embryos stained with anti-SM $\alpha$ A, the dorsal aortae were found to be completely surrounded by VSMCs (Figure 3a). The aortae of Edg1/- mice were strikingly different. SM $\alpha$ A-positive VSMCs were present on the ventral surface; however, VSMCs were deficient along the entire length of the dorsal surface examined (Figure $3 b$ ).

Transverse sections of aortae from control embryos showed two to three layers of VSMCs surrounding the 
vessel (Figure 3c). In contrast, similar sections from $E d g 1^{-/-}$embryos showed that the aortae were covered only ventrally by poorly organized SM $\alpha$ A-expressing cells (Figure $3 \mathrm{~d}$ ). SM $\alpha$ A-expressing cells were not found on the dorsal side of the mutant aortae. These results suggested initial recruitment and differentiation had taken place to produce VSMCs on the ventral side of the mutant aorta, but that the process leading to the complete envelopment of the vessel was defective. Endothelial cell morphology appeared normal in E11.5 embryos, before the onset of bleeding (Figure 3e). In the E12.5 embryos, after the onset of bleeding, the dorsal aortic surface (uncovered by VSMCs) appeared abnormal and discontinuous (Figure 3, d and f).

In E12.5 control embryos, the majority of mediumsized arteries, identified by association with SM $\alpha$ Apositive VSMCs, were surrounded by a continuous layer of VSMCs (Figure 3g). Only rare vessels were found with an incomplete covering by VSMCs. In contrast, a substantial faction of intracerebral arteries in the mutant embryos displayed a discontinuous or patchy covering by VSMCs (Figure $3 \mathrm{~h}$ ). Bleeding from these arteries was apparent by the presence of blood cells in the surrounding tissue space (Figure $3 \mathrm{~h}$ ).

The muscular layers in the gastrointestinal tract (Figure $3, i$ and $j$ ) and bronchial tree (data not shown) were well developed in the mutant embryos, indicating that there was not a generalized defect in smooth muscle.

The blood vessel defects in the $E d g 1^{-/-}$embryos were further analyzed by electron microscopy. Small blood vessels from the limb of E12.5 Edg1 $1^{-/-}$embryos illustrated a marked reduction of VSMCs/pericytes adjacent to the endothelial cells (Figure 4, a and b). The endothelial cell body was very thin, and in some areas, fragmented (data not shown). Intracerebral capillaries of mutant embryos appeared without associated microvascular pericytes (Figure 4, c and d). The endothelial cell nuclei of the mutant capillaries were abnormally rounded and enlarged. The areas surrounding these vessels were generally much less densely packed with cells. Mutant blood vessels contained normal-appearing, electron-dense interendothelial junctions (Figure 4, e and f), suggesting that endothelial cell-cell junctions formation occurred in the absence of Edg-1. Given that PDGF-B and its receptor $\beta$ are critical for the investment of capillaries with pericytes (28-30), we determined their expression in mutant embryos. We found that in $E d g 1^{-/-}$embryos PDGF-B expression was normal as determined by Western analysis and that PDGF-receptor $\beta$ was highly expressed in mesenchymal cells by immunohistochemical analysis (data not shown). Mice deficient in the transcription factor LKLF also show marked reductions in VSMCs and pericytes around vessels (31). RTPCR of E12.5 Edg1-/- RNA indicated that LKLF expression was similar to control levels (data not shown).

SPP induced migration in Edg1-/- fibroblasts. Our analysis of Edg1 embryos demonstrated a defect in blood vessel maturation that appeared to involve the ability of mural cells to properly organize and reinforce endothelial walls. Recently, Edg-1 has been implicated as the mediator of an SPP-induced migration response in different cell types (32-35). We investigated whether cells derived from the mutant embryos were defective in their migration response to SPP.

Fibroblasts were obtained from control and $E d g 1^{-/-}$ embryos. RT-PCR revealed that wild-type fibroblasts expressed transcripts for Edg1, -3 , and -5 genes (Figure 5a). Edg $1^{-/-}$fibroblasts, as expected, were devoid of the authentic Edg1 transcript but contained transcripts for Edg3 and -5. The mutant cells adhered normally to tissue culture plates and exhibited a normal mitogenic response to SPP (data not shown).

As shown in Figure 5b, 100 nM SPP induced a significant increase in the chemotaxis of wild-type fibroblasts. In contrast, the $E d g 1^{-/-}$fibroblasts did not display a significant migratory response to SPP, proving that Edg-1 is required for SPP-induced migration in these cells. Because Rac activation, which has been shown to be stimulated by SPP (24), is critical for cell migration responses (36), we compared the effect of
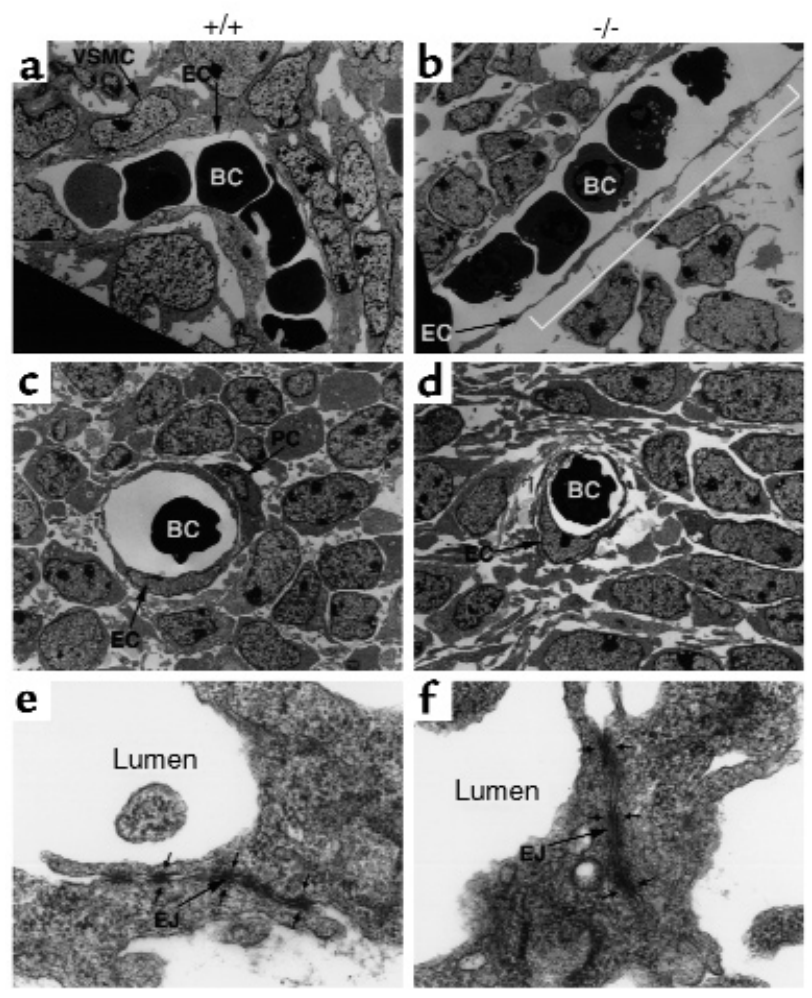

\section{Figure 4}

Reduced VSMCs and pericytes in the Edg $1^{-/-}$vessels. (a-d) EM microscopic analyses of representative small blood vessels from the limb (a and $\mathbf{b}$ ) and brain capillaries (c and d) from E12.5 wild-type and Edg1-/- embryos. Reduced number of VSMCs (bracket in $\mathbf{b}$ ) and the lack of capillary pericytes (PC) were found in the $E d g 1^{-/-}$embryos.

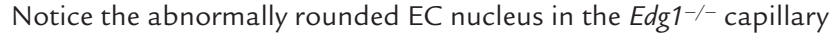
(d). (e and f) EC junctions (arrows) in wild-type and mutant embryos. Note the normal EC junction (EJ) in the Edg1-/- embryo (f). BC, blood cell. $\times 2,000$ (a-d); $\times 50,000$ (e and $\mathbf{f}$ ). 


\section{Figure 5}

Migration and Rac activation defects in Edg $1^{-/-}$embryonic fibroblasts. (a) RT-PCR analysis of Edg1, -3, and -5 expression was carried out with total RNA isolated from $E d g 1^{+/+}, E d g 1^{+/-}$, and $E d g 1^{1-}$ embryonic fibroblasts. (b) SPP chemotactic responses of embryonic fibroblasts. Serum-starved $E d g 1^{1^{+/+}}$and $E d g 1^{-/-}$fibroblasts were allowed to migrate toward a gradient produced by SPP (100 nM). Control (Cont.) indicates medium without serum was used as the chemoattractant. Chemotaxis was measured as described in Methods. Data are means \pm SD of triplicate determinations. AStatistically significant difference compared with the control, determined by Student's $t$ test $(P<0.01)$. (c) Rac activation in fibroblasts. $E d g 1^{+/+}$and $E d g 1^{-/-}$fibroblasts were serum-starved and then treated with SPP for 5 minutes. The cell lysates were used both for affinity precipitation with the PAK-1-conjugated agarose to pull down activated, GTP-bound Rac (top panel) and without fractionation to determine total Rac levels (bottom panel) by SDS-PAGE and immunoblotting. (d) Model of Edg-1 functions in blood vessel development. The Edg1 knockout demonstrates that Edg-1 is essential for vascular maturation by impairing the recruitment of smooth muscle cells to vessel walls. SPP, found in blood, may directly stimulate Edg-1 on VSMCs, facilitating their migration to vessels walls. In a second mechanism, which does not exclude the first, SPP could stimulate Edg-1 expressed on endothelial cells, which in turn recruit may VSMCs. EC, endothelial cell; SMC, smooth muscle cell.

SPP on Rac activation in wild-type and $E d g 1^{-/-}$fibroblasts (Figure 5c). In wild-type fibroblasts, SPP treatment resulted in a substantial increase in the amount of activated Rac. By contrast, no increase in activated Rac could be detected after SPP treatment of $E d g 1^{-/-}$ fibroblasts, demonstrating that Edg- 1 is required for the SPP induction of activated Rac.

\section{Discussion}

A number of receptor-mediated signaling pathways have been identified that coordinate the stages of blood vessel formation. Disruption of the genes encoding these receptors and ligands in mice has been instrumental in defining their roles (reviewed in refs. 26, 37, 38). Vasculogenesis is dependent on VEGF and its receptor tyrosine kinases, Flk-1 and Flt-1, expressed in endothelial cells (39-42). VEGF, Flk-1, and Flt-1 knockout mice die between E8.5 and E9.5 as a result of defects in the formation of the primitive vasculature. Angiogenesis and vascular remodeling require the Tie- 2 receptor tyrosine kinase on endothelial cells and its ligand, angiopoietin-1. Without Tie-2, mice have normal vasculogenesis but defective vessel sprouting, branching, and remodeling; they die at E10.5. Mice devoid of angiopoietin-1 have a similar phenotype (43-45).

Signaling pathways have also been implicated in the recruitment and differentiation of mural cells during vessel maturation. PDGF-B and its receptor- $\beta$ have been shown to be essential for the recruitment of mesenchymally derived mural cell precursors to vessel walls (30, 46). Disruption of the PDGF- $B$ or $P D G F$ receptor- $\beta$ genes in mice leads to lethal hemorrhage and edema in the perinatal stage owing to a lack of microvasculature pericytes (28-30). TGF- $\beta 1$ induces differentiation of VSMCs $\mathbf{a}$

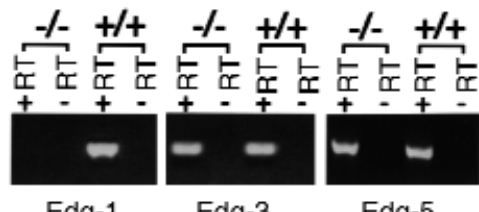

b

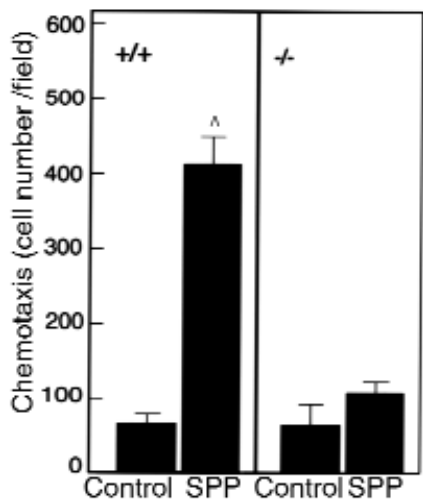

C
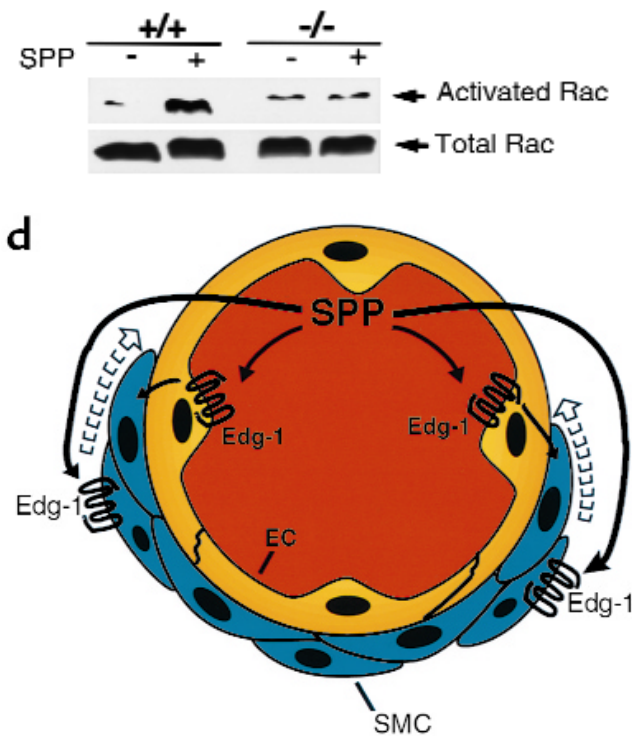

(47) after their recruitment to endothelial walls $(48,49)$. The endothelial TGF- $\beta 1$ binding protein, endoglin, and its downstream signaling molecule, SMAD5, both important in the TGF $\beta 1$ signaling pathway, also have essential roles in VSMC differentiation (50,51). Endoglinand Smad5-deficient mice die between E10.5 and E11.5 with a lack of VSMCs around major vessels.

These studies point to the critical roles played by receptor tyrosine kinases during vascular development. In contrast, the involvement of G-protein-coupled signaling pathways have not been as well characterized during development. Such pathways are important because disruption of the $G_{\alpha, 13}$ gene results in defects in embryonic vasculature formation, presumably due to a migration defect (52), However, upstream receptors involved had not been defined.

Role of Edg-1 during early vascular system development. We found that $E d g 1$ was highly expressed in the cardiovas- 
cular system during early embryonic development. Vascular endothelial cells expressed Edg1 at relatively high levels, although expression was almost exclusively found in the endothelial cells of arteries rather than of veins. A low but detectable expression was also found in VSMCs surrounding the aorta. Expression of Edg1 was prominent in cardiomyocytes, although no gross abnormalities were detected in the developing heart. In addition to the vascular system, Edg1 was found highly expressed in the developing central nervous system as has been shown previously $(16,18)$.

Severe bleeding caused lethality in Edg1/- embryos between E12.5 and E14.5. However, the mutant embryos showed a substantially normal blood vessel network when stained with antibodies to markers specific for differentiated endothelial cells such as PECAM-1 and CD34. The expression of genes known to be important for vasculogenesis and angiogenesis was not measurably affected in $E d g 1^{-1-}$ embryos. These genes included VEGF, Flk-1, Flt-1, Ang-1, and Tie-2. Each phenotype of these knockout mice is quite distinct from that of the $E d g 1^{-/-}$mice, which develop a relatively normal appearing vascular network and die between E12.5 and E14.5. These results indicate that $E d g 1$ is dispensable for vascular endothelial cell differentiation, proliferation, migration, and tube formation during vasculogenesis and for the stage of angiogenesis involving vessel sprouting and branching. The morphology and viability of endothelial cells in the mutants appeared normal until the onset of severe bleeding, suggesting that the morphology changes were secondary to the lack of supporting VSMCs and subsequent disruption of the vessels in the mutant embryos.

Although SPP signaling through Edg-1 has been shown to regulate adherens junction formation in vitro in human endothelial cells, we found no evidence of aberrant endothelial junctions in Edg1 mutant mice during development. Ultrastructural analysis of mutant endothelial cells revealed normal appearing cell-cell junctions. The phenotype of the Edg1 mutant embryos was also not in keeping with significant defects in endothelial cell junctions. Recently, it was shown that VE-cadherin, an important component of adherens junctions, controls an endothelial cell survival pathway through its intracellular interaction with $\beta$-catenin (25). Disruption of this pathway in mice resulted in impaired angiogenesis, increased endothelial apoptosis, and embryonic death by E9.5. The Edg1 mutant mice exhibited none of these characteristics. Thus the formation of functional endothelial junctions apparently proceeds normally during early development in the absence of Edg-1. This could indicate that there is functional redundancy among members of the Edg family for this process and that other Edg proteins may substitute for Edg-1. It may also indicate that the SPP-Edg regulation of adherens junction assembly is not required for blood vessel formation during development. However, this would not preclude a role for the pathway during angiogenesis in the adult.
Functions of Edg-1 during vessel maturation. In dorsal aorta, VSMC investment is initiated on the ventral side with a condensation of SM $\alpha A$-positive cells. The recruitment process continues dorsally until VSMCs have completely enveloped the endothelial tube (27). We found that the aortae of wild-type and heterozygous embryos were surrounded by several layers of elongated VSMCs. The aortae in the $E d g 1^{-/-}$embryos were strikingly abnormal in VSMC investment. Aortic sections demonstrated the presence of multiple layers of SM $\alpha$ A-positive cells, but only at the ventral surface. These results suggest that the defect in VSMC investment of vessel walls in Edg1 mutant embryos was expressed after the initial VSMC recruitment to the ventral aortic surface has taken place. This vessel abnormality was distinct from that observed in mice deficient in endoglin, the TGF- $\beta$ binding protein on endothelial cells, and in SMAD5, the TGF- $\beta 1$ signaling molecule. Both of these proteins are required for VSMC differentiation; the respective knockout mice have severely defective VSMC development with almost no SM $\alpha$ A-positive cells around vessels $(50,51)$.

In addition to a defect in mural cell recruitment in dorsal aorta, the $E d g 1^{-/-}$mice exhibited defects in the smaller vessels and in the microvasculature. By electron microscopy, we found evidence of a lack of pericytes associated with capillaries. When pericytes are deficient as in $P D G F-B^{-/-}$mice, dilated microvessels develop that are prone to rupture (53). Similarly, in the Edg1 $1^{-/}$mice, dilation of small cranial vessels and bleeding were evident.

How does Edg-1 so dramatically influence the recruitment of VSMCs to vessels during development? With fibroblasts from the Edg1 mutant mice, we found that in the absence of Edg-1, the SPP-induced activation of Rac seen in wild-type cells did not occur. Rac is a key regulator of the actin cytoskeleton and of associated activities such as cell motility $(36,54)$. Thus, the Edg1-/fibroblasts, without this signaling pathway, were unable to mount a migration response to SPP. This signaling pathway may also operate in VSMCs, and its disruption could be responsible for the defect in vascular maturation seen in the $E d g 1^{-/-}$mice. Consistent with this hypothesis, we found that Edg- 1 mediates migration of normal VSMCs toward SPP (data not shown). SPP is abundantly stored in platelets and secreted after stimulation (55). Other blood cells, including erythrocytes, neutrophils, and mononuclear cells, produce and secrete SPP constitutively (56), resulting in significant SPP levels in blood. During maturation of the dorsal aorta and possibly other vessels, plasma containing SPP could escape from immature, leaky vessels and act as a signal to recruit VSMCs that are differentiating in the proximity of vessel walls (Figure $5 \mathrm{~d}$ ).

Recently, it was shown that the zebra fish gene, mil, encodes an SPP-binding, G protein-coupled receptor of the Edg family that directs the migration of heart precursors to the midline during embryonic development (57). Interestingly, mil does not function in the 
migrating precursor cells, but in the paraxial cells at the midline, presumably by creating a permissive environment for migration. Sequence and functional similarities suggest that mil may be the ortholog of the mammalian $E d g 5$ gene. However, the indirect influence of mil on migrating cells, as well as the high level of expression of Edg1 in arterial endothelial cells, raises the possibility that Edg-1 stimulation on endothelial cells may regulate the recruitment of VSMCs (Figure $5 \mathrm{~d}$ ). This might occur via the upregulation of adhesion molecules on endothelial cells, or by stimulating the secretion of recruitment factors for VSMCs. Further studies using tissue-specific knockouts of Edg1 will be required to address precisely how Edg-1 functions. Nevertheless, our data demonstrate an indispensable role of Edg1 in vascular maturation and, together with the results showing mil is essential for heart organogenesis, indicate that different members of the Edg receptor family regulate distinct aspects of cardiovascular development through sphingolipid signaling pathways.

In summary, we have uncovered a unique and vital role for the $\mathrm{G}$ protein-coupled receptor, Edg-1, in blood vessel formation during development. The SPP-Edg signaling pathway may also have important functions in adult vascular biology. SPP stimulation of Edg receptors on endothelial cells in vitro results in proliferation, migration, and tube formation $(24,32,58)$, all requisite for the angiogenic process. Thus, the release of SPP after platelet activation, with subsequent stimulation of the Edg receptors on both endothelial cells and VSMCs, may drive blood vessel formation during wound healing and solid tumor growth $(59,60)$. If so, the SPP-Edg signaling pathway is a potential target for therapeutic manipulation during these and other processes that are dependent on angiogenesis.

\section{Acknowledgments}

We thank J. Van Brocklyn for help in the early stages of this project and A. Howard for producing the figures.

1. Shayman, J.A. 2000. Perspectives in basic science: sphingolipids. Kidney Int. 58:11-26.

2. Huwiler, A., Kolter, T., Pfeilschifter, J., and Sandhoff, K. 2000. Physiology and pathophysiology of sphingolipid metabolism and signaling. Biochim. Biophys. Acta. 1485:63-99.

3. Spiegel, S., and Merrill, A.H., Jr. 1996. Sphingolipid metabolism and cell growth regulation. FASEB J. 10:1388-1397.

4. Lee, M.J., et al. 1998. Sphingosine-1-phosphate as a ligand for the G protein-coupled receptor EDG-1. Science. 279:1552-1555.

5. Hla, T., and Maciag, T. 1990. An abundant transcript induced in differentiating human endothelial cells encodes a polypeptide with structural similarities to G-protein-coupled receptors. J. Biol. Chem. 265:9308-9313.

6. Goetzl, E.J., and An, S. 1998. Diversity of cellular receptors and functions for the lysophospholipid growth factors lysophosphatidic acid and sphingosine 1-phosphate. FASEB J. 12:1589-1598.

7. Spiegel, S., and Milstien, S. 2000. Functions of a new family of sphingosine-1-phosphate receptors. Biochim. Biophys. Acta. 1484:107-116.

8. Hla, T., et al. 1999. Sphingosine-1-phosphate: extracellular mediator or intracellular second messenger? Biochem. Pharmacol. 58:201-207.

9. Chun, J., Contos, J.J., and Munroe, D. 1999. A growing family of receptor genes for lysophosphatidic acid (LPA) and other lysophospholipids (LPs). Cell Biochem. Biophys. 30:213-242.

10. Moolenaar, W.H. 1999. Bioactive lysophospholipids and their G protein-coupled receptors. Exp. Cell. Res. 253:230-238.

11. Lee, M.J., Evans, M., and Hla, T. 1996. The inducible G protein-cou- pled receptor edg-1 signals via the G(i)/mitogen-activated protein kinase pathway. J. Biol. Chem. 271:11272-11279.

12. Ancellin, N., and Hla, T. 1999. Differential pharmacological properties and signal transduction of the sphingosine 1-phosphate receptors EDG-1, EDG-3, and EDG-5. J. Biol. Chem. 274:18997-19002.

13. An, S., Bleu, T., and Zheng, Y. 1999. Transduction of intracellular calcium signals through $\mathrm{G}$ protein-mediated activation of phospholipase C by recombinant sphingosine 1-phosphate receptors. Mol. Pharmacol. 55:787-794.

14. Windh, R.T., et al. 1999. Differential coupling of the sphingosine 1phosphate receptors Edg-1, Edg-3, and H218/Edg-5 to the g(i), g(q), and $\mathrm{G}(12)$ families of heterotrimeric G proteins. J. Biol. Chem. 274:27351-27358.

15. Hecht, J.H., Weiner, J.A., Post, S.R., and Chun, J. 1996. Ventricular zone gene-1 (vzg-1) encodes a lysophosphatidic acid receptor expressed in neurogenic regions of the developing cerebral cortex. $J$. Cell. Biol. 135:1071-1083.

16. Liu, C.H., and Hla, T. 1997. The mouse gene for the inducible G-protein-coupled receptor edg-1. Genomics. 43:15-24.

17. Graler, M.H., Bernhardt, G., and Lipp, M. 1998. EDG6, a novel G-protein-coupled receptor related to receptors for bioactive lysophospholipids, is specifically expressed in lymphoid tissue. Genomics. 53:164-169.

18. Zhang, G., Contos, J.J., Weiner, J.A., Fukushima, N., and Chun, J. 1999. Comparative analysis of three murine G-protein coupled receptors activated by sphingosine-1-phosphate. Gene. 227:89-99.

19. Nehls, M., et al. 1996. Two genetically separable steps in the differentiation of thymic epithelium. Science. 272:886-889.

20. Mountford, P.S., and Smith, A.G. 1995. Internal ribosome entry sites and dicistronic RNAs in mammalian transgenesis. Trends Genet. 11:179-184.

21. Liu, Y., et al. 1999. A genetic model of substrate deprivation therapy for a glycosphingolipid storage disorder. J. Clin. Invest. 103:497-505.

22. Wang, F., Nohara, K., Olivera, A., Thompson, E.W., and Spiegel, S. 1999. Involvement of focal adhesion kinase in inhibition of motility of human breast cancer cells by sphingosine 1-phosphate. Exp. Cell Res. 247:17-28.

23. Benard, V., Bohl, B.P., and Bokoch, G.M. 1999. Characterization of $\mathrm{rac}$ and $\mathrm{cdc} 42$ activation in chemoattractant-stimulated human neutrophils using a novel assay for active GTPases. J. Biol. Chem. 274:13198-13204.

24. Lee, M.J., et al. 1999. Vascular endothelial cell adherens junction assembly and morphogenesis induced by sphingosine-1-phosphate. Cell. 99:301-312.

25. Carmeliet, P., et al. 1999. Targeted deficiency or cytosolic truncation of the VE-cadherin gene in mice impairs VEGF-mediated endothelial survival and angiogenesis. Cell. 98:147-157.

26. Carmeliet, P. 2000. Mechanisms of angiogenesis and arteriogenesis. Nat. Med. 6:389-395.

27. Takahashi, Y., Imanaka, T., and Takano, T. 1996. Spatial and temporal pattern of smooth muscle cell differentiation during development of the vascular system in the mouse embryo. Anat. Embryol. (Berl.) 194:515-526.

28. Leveen, P., et al. 1994. Mice deficient for PDGF B show renal, cardiovascular, and hematological abnormalities. Genes Dev. 8:1875-1887.

29. Soriano, P. 1994. Abnormal kidney development and hematological disorders in PDGF beta-receptor mutant mice. Genes Dev. 8:1888-1896

30. Lindahl, P., Johansson, B.R., Leveen, P., and Betsholtz, C. 1997. Pericyte loss and microaneurysm formation in PDGF-B-deficient mice. Science. 277:242-245.

31. Kuo, C.T., et al. 1997. The LKLF transcription factor is required for normal tunica media formation and blood vessel stabilization during murine embryogenesis. Genes Dev. 11:2996-3006.

32. Wang, F., et al. 1999. Sphingosine 1-phosphate stimulates cell migration through a G(i)-coupled cell surface receptor. Potential involvement in angiogenesis. J. Biol. Chem. 274:35343-35350.

33. Panetti, T.S., Nowlen, J., and Mosher, D.F. 2000. Sphingosine-1-phosphate and lysophosphatidic acid stimulate endothelial cell migration. Arterioscler. Thromb. Vasc. Biol. 20:1013-1019.

34. English, D., et al. 1999. Induction of endothelial cell chemotaxis by sphingosine 1-phosphate and stabilization of endothelial monolayer barrier function by lysophosphatidic acid, potential mediators of hematopoietic angiogenesis. J. Hematother. Stem Cell Res. 8:627-634.

35. Kon, J., et al. 1999. Comparison of intrinsic activities of the putative sphingosine 1-phosphate receptor subtypes to regulate several signaling pathways in their cDNA-transfected Chinese hamster ovary cells. J. Biol. Chem. 274:23940-23947.

36. Hall, A. 1998. Rho GTPases and the actin cytoskeleton. Science. 279:509-514

37. Folkman, J., and D'Amore, P.A. 1996. Blood vessel formation: what is its molecular basis? Cell. 87:1153-1155. 
38. Hanahan, D. 1997. Signaling vascular morphogenesis and maintenance. Science. 277:48-50

39. Carmeliet, P., et al. 1996. Abnormal blood vessel development and lethality in embryos lacking a single VEGF allele. Nature. 380:435-439.

40. Ferrara, N., et al. 1996. Heterozygous embryonic lethality induced by targeted inactivation of the VEGF gene. Nature. 380:439-442.

41. Shalaby, F., et al. 1995. Failure of blood-island formation and vasculogenesis in Flk-1-deficient mice. Nature. 376:62-66.

42. Fong, G.H., Rossant, J., Gertsenstein, M., and Breitman, M.L. 1995 Role of the Flt-1 receptor tyrosine kinase in regulating the assembly of vascular endothelium. Nature. 376:66-70.

43. Dumont, D.J., et al. 1994. Dominant-negative and targeted null mutations in the endothelial receptor tyrosine kinase, tek, reveal a critical role in vasculogenesis of the embryo. Genes Dev. 8:1897-1909.

44. Sato, T.N., et al. 1995. Distinct roles of the receptor tyrosine kinases Tie- 1 and Tie- 2 in blood vessel formation. Nature. 376:70-74.

45. Suri, C., et al. 1996. Requisite role of angiopoietin-1, a ligand for the TIE2 receptor, during embryonic angiogenesis. Cell. 87:1171-1180.

46. Hellstrom, M., Kal, M., Lindahl, P., Abramsson, A., and Betsholtz, C. 1999. Role of PDGF-B and PDGFR-beta in recruitment of vascula smooth muscle cells and pericytes during embryonic blood vessel formation in the mouse. Development. 126:3047-3055.

47. Shah, N.M., Groves, A.K., and Anderson, D.J. 1996. Alternative neural crest cell fates are instructively promoted by TGFbeta superfamily members. Cell. 85:331-343.

48. Hirschi, K.K., Rohovsky, S.A., Beck, L.H., Smith, S.R., and D'Amore, P.A. 1999. Endothelial cells modulate the proliferation of mural cell precursors via platelet-derived growth factor-BB and heterotypic cell contact. Circ. Res. 84:298-305.

49. Hirschi, K.K., Rohovsky, S.A., and D'Amore, P.A. 1998. PDGF, TGFbeta, and heterotypic cell-cell interactions mediate endothelial cell- induced recruitment of $10 \mathrm{~T} 1 / 2$ cells and their differentiation to a smooth muscle fate [erratum, 1998. 141:1287]. J. Cell Biol, 141:805-814.

50. Li, D.Y., et al. 1999. Defective angiogenesis in mice lacking endoglin. Science. 284:1534-1537.

51. Yang, X., et al. 1999. Angiogenesis defects and mesenchymal apoptosis in mice lacking SMAD5. Development. 126:1571-1580.

52. Offermanns, S., Mancino, V., Revel, J.P., and Simon, M.I. 1997. Vascular system defects and impaired cell chemokinesis as a result of Galpha13 deficiency. Science. 275:533-536.

53. Lindahl, P., Hellstrom, M., Kalen, M., and Betsholtz, C. 1998. Endothelial-perivascular cell signaling in vascular development: lessons from knockout mice. Curr. Opin. Lipidol. 9:407-411.

54. Nobes, C.D., and Hall, A. 1995. Rho, rac, and cdc42 GTPases regulate the assembly of multimolecular focal complexes associated with actin stress fibers, lamellipodia, and filopodia. Cell. 81:53-62.

55. Yatomi, Y., Ruan, F., Hakomori, S., and Igarashi, Y. 1995. Sphingosine-1-phosphate: a platelet-activating sphingolipid released from agonist-stimulated human platelets. Blood. 86:193-202.

56. Yang, L., Yatomi, Y., Miura, Y., Satoh, K., and Ozaki, Y. 1999. Metabolism and functional effects of sphingolipids in blood cells. $\mathrm{Br}$. J. Haematol. 107:282-293.

57. Kupperman, E., An, S., Osborne, N., Waldron, S., and Stainier, D.Y.R. 2000. A sphingosine-1-phosphate receptor regulates cell migration during vertebrate heart development. Nature. 406:192-195.

58. Lee, O., et al. 1999. Sphingosine 1-phosphate induces angiogenesis: its angiogenic action and signaling mechanism in human umbilical vein endothelial cells. Biochem. Biophys. Res. Commun. 264:743-750.

59. Folkman, J. 1995. Angiogenesis in cancer, vascular, rheumatoid and other disease. Nat. Med. 1:27-31.

60. Pinedo, H.M., Verheul, H.M., D’Amato, R.J., and Folkman, J. 1998. Involvement of platelets in tumour angiogenesis? Lancet. 352:1775-1777. 